\title{
Computational approaches for exploring the potential synergy and polypharmacology of Duhuo Jisheng Decoction in the therapy of osteoarthritis
}

\author{
CHUN-SONG ZHENG ${ }^{1,2}$, XIAO-JIE XU ${ }^{1,3}$, HONG-ZHI YE ${ }^{1,2}$, GUANG-WEN \\ $\mathrm{WU}^{2}, \mathrm{XI-HAI} \mathrm{LI}^{2}$, SU-PING HUANG ${ }^{1}$ and XIAN-XIANG LIU ${ }^{1}$ \\ ${ }^{1}$ Fujian Academy of Integrative Medicine; ${ }^{2}$ Fujian Key Laboratory of Integrative Medicine on Geriatrics, \\ Fujian University of Traditional Chinese Medicine, Fuzhou 350122; ${ }^{3}$ College of Chemistry and Molecular Engineering, \\ Peking University, Beijing 100871, P.R. China
}

Received December 1, 2012; Accepted March 26, 2013

DOI: $10.3892 / \mathrm{mmr} .2013 .1411$

\begin{abstract}
The Duhuo Jisheng Decoction (DHJSD), a traditional Chinese medicine (TCM), has been widely used to treat osteoarthritis (OA). However, the mechanisms of action of the DHJSD have not been clearly elucidated. In the present study, the compounds in the DHJSD were characterized by three computational methods; the ligand clustering, chemical space distribution and network construction and analysis methods. The compounds that formed the medical composition of the DHJSD were divided into 10 clusters and possessed a broad diversity in chemical space distribution. The compounds also had the same coverage of chemical space as the OA drug/drug-like compounds from DrugBank. In addition, multiple active compounds were identified as able to target multiple proteins in the drug-target association networks (D-T networks). A certain number of key compounds in the D-T networks have been previously reported in the literature. The present study also constructed drug-drug association networks (D-D networks) and classified the DHJSD compounds into five clusters. The clusters represented multiple diverse combinations binding to the OA targets. These results suggested that
\end{abstract}

Correspondence to: Dr Xian-Xiang Liu, Academy of Integrative Medicine, Fujian University of Traditional Chinese Medicine, 1 Huatuo Road, Minhou Shangjie, Fuzhou, Fujian 350122, P.R. China

E-mail: liuxianxiang@163.com

Abbreviations: ADAMTS-4, Aggrecanase-1; MMP, matrix metalloproteinase; TNF- $\alpha$, tumor necrosis factor $\alpha$; iNOS, inducible nitric oxide synthase; COX, cyclooxygenase; CDK, cyclin-dependent kinase; HO, heme oxygenase; PPAR $\gamma$, peroxisome proliferator activated receptor- $\gamma$; GSK- $3 \beta$, glycogen synthase kinase-3 $\beta$; ER, estrogen receptor; VDR, vitamin D nuclear receptor; TGF- $\beta 1$, transforming growth factor- $\beta$ type I; Std Dev, standard deviation

Key words: Duhuo Jisheng Decoction, osteoarthritis, chemical space, virtual screening, network analysis, polypharmacology the DHJSD had drug- and lead-like compounds with potential synergy and polypharmacology against OA.

\section{Introduction}

Osteoarthritis (OA) is the most common cause of chronic disability in older adults and is a progressive and multifactorial disease characterized by cartilage degeneration, osteophytes and synovial fibrosis. To date, pharmacological management for the majority of OA patients has targeted the symptoms of the disease rather than the underlying cause and pathophysiology (1). This means that there are no satisfactory pharmacological therapies for OA. The main drugs on the market for OA treatment are analgesics and non-steroidal anti-inflammatory drugs. These drugs mainly alleviate the symptoms but have numerous side-effects and poor cost-effectiveness (2). The requirement to develop new and relatively inexpensive treatment strategies for $\mathrm{OA}$, which are able to restore full function or return the tissue to its native normal state, has gained medical acknowledgement.

At present, several lines of evidence suggest that the traditional notion of 'one drug-one protein' for one disease is no longer applicable and that treatment for the most complex diseases may best be attempted using polypharmacological approaches $(3,4)$. The model for drug discovery must be transformed from a single target-based to system biology-oriented one (4). Herbal remedies are the most prevalent and effective treatment in the management of chronic illnesses in a number of Asian countries (5). The synergistic actions of the herbal ingredients may be responsible for this therapeutic efficacy (6). In addition, numerous herbs have shown potential anti-inflammatory mechanisms in a variety of clinical trials. Extracts from these herbs may be able to alleviate OA symptoms and incur fewer side effects $(7,8)$. Thus, it is important to elucidate the synergy and polypharmacology of traditional Chinese medicine (TCM) to aid the identification of new OA drugs.

The Duhuo Jisheng Decoction (DHJSD), a TCM from Bei Ji Qian Jin Yao Fang developed by Sun SM in the Tang Dynasty (9), has been prescribed to treat OA in China. The 
results of animal experiments showed that DHJSD was able to decrease the levels of tumor necrosis factor (TNF)- $\alpha$ and matrix metalloproteinase (MMP)-3 in the serum and synovial fluid of rabbits with OA (10). The results of clinical experiments showed that DHJSD reduced the levels of TNF- $\alpha$ and high-sensitivity $\mathrm{C}$-reactive protein (hs-CRP) in the synovial joints in patients with knee OA (11). Clinical observations also suggested that the DHJSD was able to reduce pain, stiffness and improve physical functions (12). To the best of our knowledge, however, the underlying mechanisms of the DHJSD in the management of OA are not fully understood. Therefore, the aim of the present study was to identify the synergy and polypharmacology of the DHJSD, using well-known computational methods $(4,13)$, in order to obtain a general method to benefit modern TCM development.

The flow chart of the computational design in the present study is shown in Fig. 1.

\section{Materials and methods}

Ligand preparation and clustering. DHJSD consists of 15 species of medicinal herbs: Angelica pubescens, Saposhnikovia divaricata, Ligusticum chuanxiong, Achyranthes bidentata, Loranthus parasiticus, Gentiana macrophylla, Eucommia ulmoides, Angelicae sinensis, Poria cocos, Codonopsis pilosula, Radix rehmanniae preparata, Radix paeoniae alba, Asarum sieboldii, Glycyrrhiza uralensis and Cinnamomum cassia. The compounds identified in the medicinal herbs of the DHJSD were collected from the Chinese Herbal Drug Database and the Handbook of the Constituents in Chinese Herb Original Plants (14,15). Subsequent to excluding the duplicates, the total number of compounds obtained was 496. All the compounds in the collection were converted into three-dimensional structures and energy optimizations were performed using the Discovery Studio 2.0 (DS 2.0) software (Accelrys Inc., San Diego, CA, USA) based on the Merck molecular forcefield (MMFF). The protocol for the cluster ligands method was then used to cluster the compounds from the DHJSD (16).

Molecular descriptors calculation. Chemical space is a generalized data visualization method used to describe the well-known belief among chemists that similar compounds may have similar properties (17). Prior to virtual screening, the chemical space of the compounds from the DHJSD was studied. Chemical space was defined by calculating a given set of descriptors for each molecule and using these values as coordinates in the multi-dimensional space (18). The protocol to calculate the molecular properties in the quantitative structure-activity relationship (QSAR) module of DS 2.0 was introduced to characterize the compounds from the DHJSD and their chemical space was constructed using 150 diversity descriptors, including the molecular properties in 1-, 2- and 3-dimensions. Principal component analysis (PCA) was then performed to map the distribution of the compounds in chemical space.

Virtual screening. LigandFit, a modern docking program within DS 2.0 was used for each run. The three-dimensional crystal structures of $20 \mathrm{key}$ proteins that were associated with OA (19) were selected as the docking receptors from
Table I. 20 key proteins related to OA.

\begin{tabular}{ll}
\hline Protein & PDB code \\
\hline ADAMTS-4 & $2 \mathrm{RJP}$ \\
TNF- $\alpha$ & $2 \mathrm{AZ5}$ \\
iNOS & $2 \mathrm{Y} 37$ \\
COX-1 & $3 \mathrm{NT} 1$ \\
COX-2 & $3 \mathrm{~N} 8 \mathrm{X}$ \\
MMP-1 & $966 \mathrm{C}$ \\
MMP-8 & $1 \mathrm{ZS} 0$ \\
MMP-9 & $1 \mathrm{GKC}$ \\
MMP-11 & $1 \mathrm{HV} 5$ \\
MMP-12 & $3 \mathrm{RTS}$ \\
MMP-13 & $3 \mathrm{I}$ I \\
Caspase-3 & $2 \mathrm{CNN}$ \\
Caspase- 8 & $1 \mathrm{QDU}$ \\
CDK2 & $3 \mathrm{PXY}$ \\
HO-1 & $3 \mathrm{TGM}$ \\
PPAR $\gamma$ & $2 \mathrm{VSR}$ \\
GSK-3 $\beta$ & $3 \mathrm{~L} 1 \mathrm{~S}$ \\
ER & $1 \mathrm{UOM}$ \\
VDR & $1 \mathrm{DB} 1$ \\
TGF- $\beta 1$ & $1 \mathrm{RW} 8$ \\
\hline
\end{tabular}

OA, osteoarthritis; PDB, protein data bank; ADAMTS-4, Aggrecanase-1; MMP, matrix metalloproteinase; $\mathrm{TNF}-\alpha$, tumor necrosis factor $\alpha$; iNOS, inducible nitric oxide synthase; COX, cyclooxygenase; CDK, cyclin-dependent kinase; HO, heme oxygenase; PPAR $\gamma$, peroxisome proliferator-activated receptor- $\gamma$; GSK- $3 \beta$, glycogen synthase kinase-3 $\beta$; ER, estrogen receptor; VDR, vitamin D nuclear receptor; TGF- $\beta 1$, transforming growth factor- $\beta$ type I

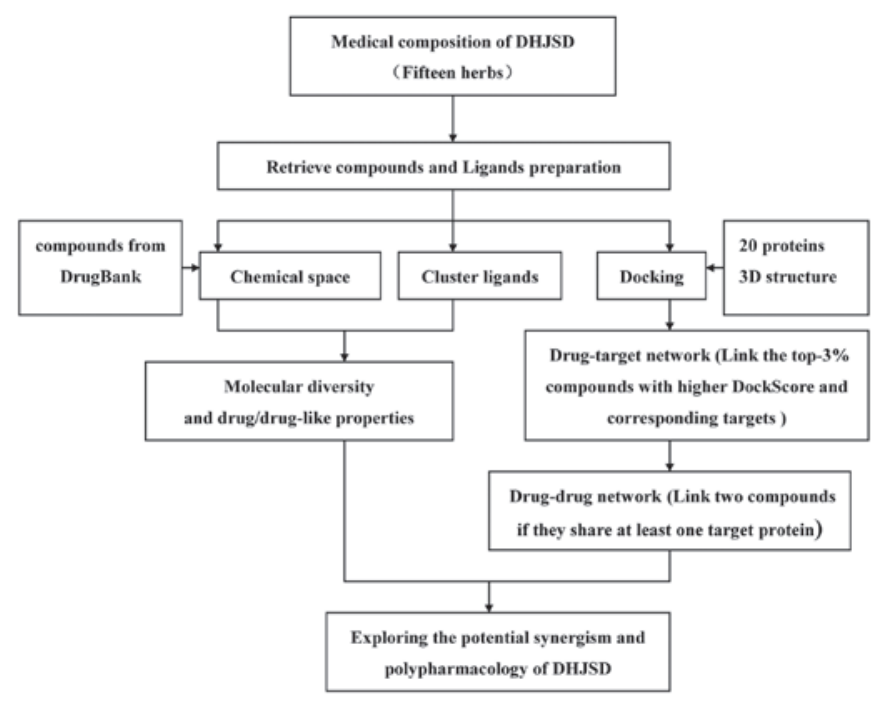

Figure 1. Flow chart of the computational design in the present study. DHJSD, Duhuo Jisheng Decoction.

the RCSB Protein Data Bank (PDB; Table I). All crystallographic waters were removed from the file and the hydrogen atoms were added. The inhibitor from the PDB file was used to define the active site. A total of 496 compounds from the 
Table II. Mean, standard deviation, minimum and maximum values of the key molecular descriptors of the DHJSD compounds.

\begin{tabular}{|c|c|c|c|c|}
\hline Name & Mean & Std dev & Minimum & Maximum \\
\hline Molecular weight & 331.51 & 187.99 & 59.11 & 1013.13 \\
\hline Number of hydrogen acceptors & 5.45 & 5.21 & 0 & 26 \\
\hline Number of hydrogen donors & 2.74 & 3.11 & 0 & 17 \\
\hline ALogP & 2.44 & 3.26 & -9.55 & 13.60 \\
\hline Molecular solubility & -3.73 & 2.94 & -15.01 & 1.92 \\
\hline Number of rotatable bonds & 5.33 & 4.94 & 0 & 32 \\
\hline Nitrogen count & 0.11 & 0.63 & 0 & 8 \\
\hline Oxygen count & 5.41 & 5.20 & 0 & 26 \\
\hline
\end{tabular}

DHJSD, Duhuo Jisheng Decoction; Std dev, standard deviation.

DHJSD were docked into the protein models. The DockScore evaluated the ligand position and orientation based on the most favorable energy production from the interactions between the ligand conformations and receptor proteins (20). All 496 docked structures were thus sorted according to their DockScore.

Network construction and analysis. Cytoscape 2.8.3 was carried out to construct the subsequent networks (21). According to the DockScore sorting of the compounds, the top 14 compounds (the top 3\%) were selected (22). These compounds and the corresponding target proteins were regarded as network nodes. Therefore, drug-target association networks (D-T networks) were constructed by linking the compound and proteins. If two compounds shared $\geq 1$ target proteins, they were linked to map the drug-drug association networks (D-D networks). Data were analyzed using Cytoscape plugins.

\section{Results}

Clustering of the DHJSD compounds. Clustering was used to group the DHJSD compounds by employing the default settings of the cluster ligands method. In total, 10 compounds with maximum dissimilarity were selected as the cluster centers and the other compounds were attached to their clusters according to the cluster assignment and their properties with regard to the distance to the center. The distribution of the various clusters (Fig. 2) suggested that the DHJSD may be considered a natural combinatorial chemical library with molecular diversity.

Distribution of the DHJSD compounds in chemical space. Fig. 3A shows the global property map of chemical space, starting from 1-, 2-and 3-dimensional-based descriptors of the DHJSD compounds. The DHJSD compounds possessed a broad diversity in chemical space. Table II shows that the majority of the DHJSD compounds had good drug-like properties according to Lipinski's rule of five (23). To confirm the drug-like properties of the DHJSD compounds, the chemical space distribution of the known drug/drug-like compounds for OA from DrugBank was also charted (24). The overlap fraction (Fig. 3B) represented the drug/drug-like chemical space,
Table III. Network properities of the D-T and D-D networks.

\begin{tabular}{lcc}
\hline Parameters & D-T network & D-D network \\
\hline Network density & 0.027 & 0.169 \\
Network heterogeneity & 1.185 & 0.568 \\
Network centralization & 0.072 & 0.39 \\
Characteristic path length & 4.138 & 2.204 \\
Mean number of neighbors & 3.875 & 20.839 \\
Shortest paths, n (\%) & $20592(100)$ & $15252(100)$ \\
Cluster coefficient & 0 & 0.829 \\
\hline
\end{tabular}

D-T network, drug-target association network; D-D network, drug-drug association network.

indicating that the nature of the DHJSD compounds was extremely similar to that of the drug/drug-like compounds.

Investigation of the multi-target compounds of the DHJSD. Screening of the DHJSD compounds and their targets was performed to generate a bipartite graph of the compound-protein interactions in which a compound and a protein were connected to each other if the protein was an action target of the drug, thus leading to a D-T network (Fig. 4). The histogram of the number of targets associated with each compound is shown in Fig. 5. The majority of compounds acted on only one target, but a small number of compounds acted on a large number of targets, up to a maximum of 14 . The general network properties and key compounds of the D-T networks are listed in Tables III and IV, respectively.

Combination therapy of the DHJSD for OA. In the D-D network (Fig. 6), the nodes represented the compounds, two of which were connected to each other if they shared at least one target protein. The general network properties of the D-D networks are listed in Table III. MCODE, a Cytoscape plugin, was used to identify five clusters in the D-D networks (Fig. 7; Table V) and to show the various compounds in different types of networks and the various clusters with their effect targets. This revealed the combination therapy mechanism of the DHJSD for OA. 
Table IV. Top-10 key compounds in the D-T network that acted on the largest number (degree) of targets.

\begin{tabular}{|c|c|c|c|}
\hline Index & Known & Chemical name & Degree \\
\hline 65 & Yes & Chlorogenic acid & 14 \\
\hline 59 & Yes & Avicularin & 12 \\
\hline 152 & Yes & Macrophylloside D & 10 \\
\hline 74 & No & Erytho-dihydroxydehydrodiconiferyl alcohol & 10 \\
\hline 151 & Yes & Macrophylloside C & 9 \\
\hline 148 & No & 6'-O- $\beta$-D-Glucosyl Gentiopicroside & 9 \\
\hline 95 & No & Methyl chlorogenate & 8 \\
\hline 222 & No & Folic acid & 6 \\
\hline 31 & No & Marmesin & 6 \\
\hline 82 & Yes & Geniposidic acid & 5 \\
\hline
\end{tabular}

D-T, drug-target.

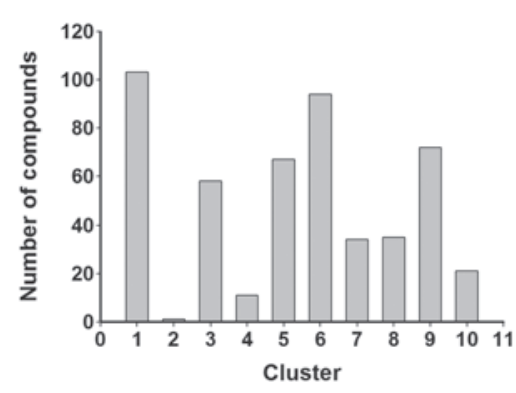

Figure 2. Clustering distribution of the DHJSD compounds. DHJSD, Duhuo Jisheng Decoction.

\section{Discussion}

OA is much more complex than initially anticipated as it is often caused by multiple molecular abnormalities, rather than being the result of a single effect (25). In this regard, the application of combinational drugs or multi-target drugs, in which $\geq 2$ drugs interact with multiple targets simultaneously, may be considered as a rational and effective treatment strategy for OA. Notably, TCM is a holistic approach to health that attempts to bring the body, mind and spirit into harmony, thus it advocates drug-combined administrations (26). Several studies have demonstrated that Chinese medicinal herbs have the potential to ameliorate the progress of OA and such herbs have received increasing attention $(7,8)$.

DHJSD, an extremely common TCM, has been used in the treatment of patients with OA in China. In the present study, a system using the compounds in the original plants of the DHJSD were chosen. The compounds were classified into 10 groups and possessed a broader diversity in chemical space. The corresponding means of the molecular descriptors, including the molecular weight, number of hydrogen acceptors, number of hydrogen donors and ALogP were 331.51, 5.45, 2.74 and 2.44 , respectively. This showed that the majority of the compounds satisfied Lipinski's rule of five. Moreover, common chemical space was observed between the two compounds sets from the DHJSD and DrugBank. Any overlap was extremely likely to contain active compounds and synergistic therapeutic
$\mathbf{A}$

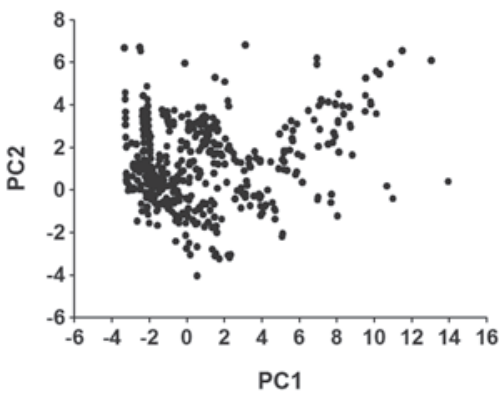

B

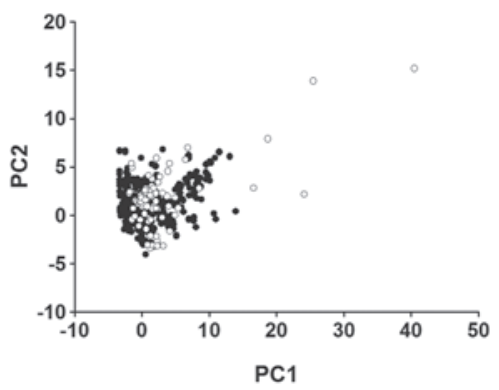

Figure 3. Distribution of the first two principal components of the DHJSD and drug/drug-like compounds in chemical space. The black and white circles represent the chemical space of the DHJSD compounds and drug/drug-like compounds, respectively. (A) Global property map of chemical space. (B) The overlap fraction represented the drug/drug-like chemical space, indicating that the nature of the DHJSD compounds was extremely similar to that of the drug/drug-like compounds. DHJSD, Duhuo Jisheng Decoction.

actions, therefore, the present study used drug-target interaction model experiments to aid in clarifying the effect of the DHJSD compounds on OA.

A virtual screening study was performed in the search for the DHJSD compounds with the potential to inhibit targets related to OA. The top $3 \%$ of the compounds with the highest DockScores and the corresponding target proteins were linked to construct the D-T networks, which were used to search for multi-target compounds (promiscuous drugs) in the DHJSD. The results demonstrated that the DHJSD is a broad-spectrum recipe inhibiting a number of significant target proteins. Each compound from the DHJSD was correlated with 2.26 targets 


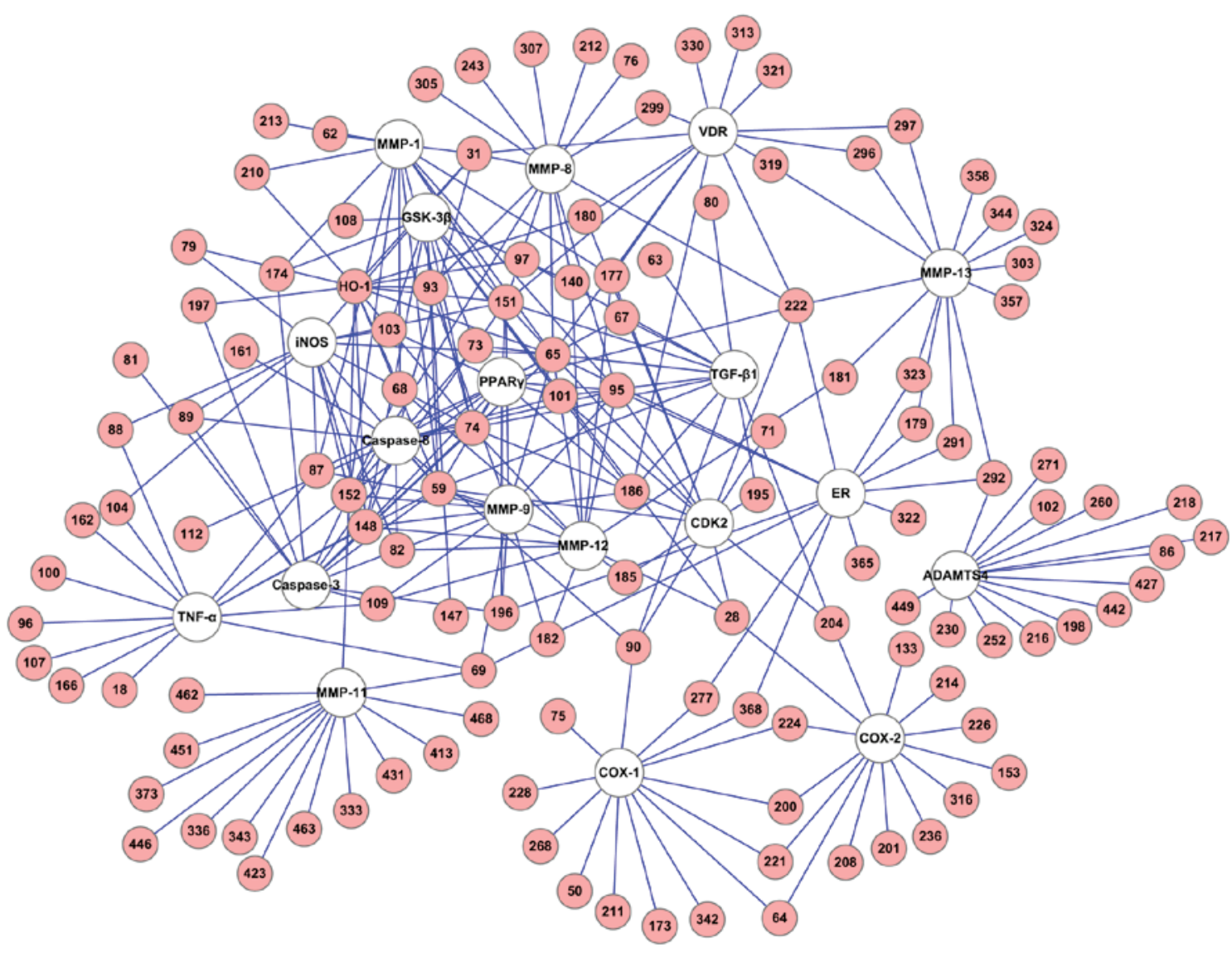

Figure 4. Drug-target association network (D-T network). The white and pink circles represent the target proteins associated with OA and the DHJSD compounds, respectively. OA, osteoartritis; DHJSD, Duhuo Jisheng Decoction.

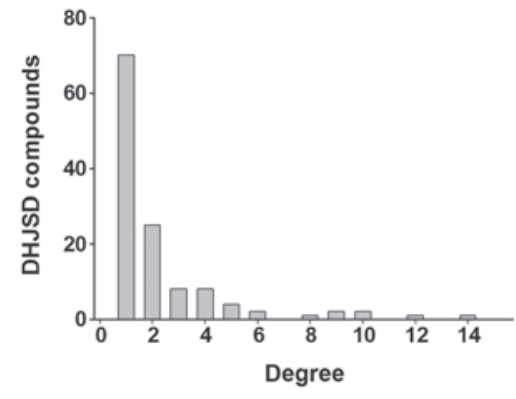

Figure 5. Distribution of the number of targets associated with each compound. DHJSD, Duhuo Jisheng Decoction.

on average and the maximum number of targets a compound acted on was 14. Table III shows the biological activities of certain compounds that have been reported in the literature (27-30). Investigators have shown that multi-targeted or 'promiscuous' drugs may often more effective than precisely targeted drugs, particularly in complex diseases (31). This may be one reason to explain for DHJSD being effective in curing OA.

To gain an improved understanding of the functions of the DHJSD, the D-D network was contructed and five clusters were identified that were used to classify the DHJSD compounds into varying network types. Each cluster represented a separate multi-drug combination and function. The botanical sources of each cluster also varied (Table V). The compounds in cluster 1 were associated with 17 target proteins

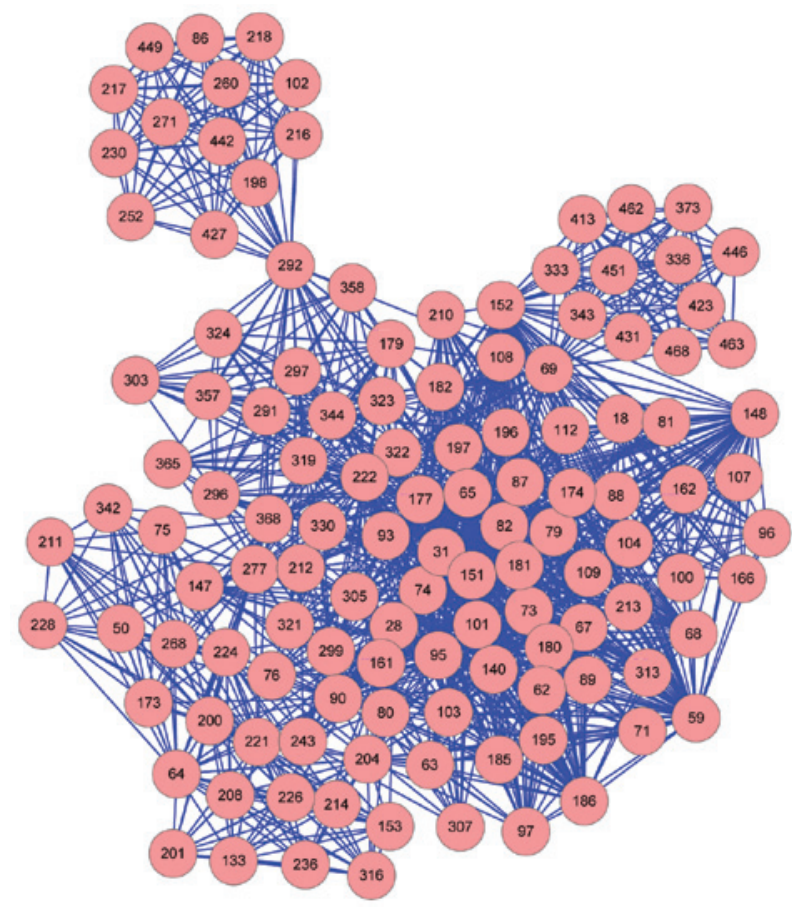

Figure 6. The drug-drug association network (D-D network).

and the compounds in cluster 2 had interactions with 15 target proteins. The compounds in the two clusters interacted with 14 common target proteins. The results indicated that the 
Table V. Information related to the categories of the subnetworks.

\begin{tabular}{|c|c|c|}
\hline Cluster & Effect targets & Botanical sources of compounds \\
\hline 1 & $\begin{array}{l}\text { MMP-1, MMP-8, MMP-9, MMP-11, } \\
\text { MMP-12, PPAR } \gamma, \text { GSK-3 } \beta, \text { VDR, } \\
\text { iNOS, ER, Caspase-3, Caspase- } 8 \text {, } \\
\text { CDK2, HO-1, TGF- } \beta 1, \text { COX-1, TNF- } \alpha\end{array}$ & $\begin{array}{l}\text { Angelica pubescens, Saposhnikovia divaricata, Ligusticum chuanxiong, } \\
\text { Achyranthes bidentata, Loranthus parasiticus, Gentiana macrophylla, } \\
\text { Eucommia ulmoides, Poria cocos, Codonopsis pilosula, } \\
\text { Radix rehmanniae preparata, Radix paeoniae alba, Glycyrrhiza uralensis, } \\
\text { Cinnamomum cassia }\end{array}$ \\
\hline 2 & $\begin{array}{l}\text { MMP-1, MMP-8, MMP-9, MMP-12, } \\
\text { MMP- } 13, \text { PPAR } \gamma, \text { GSK-3 } \beta, \text { VDR, } \\
\text { iNOS, ER, Caspase-3, CDK2, HO-1, } \\
\text { TGF- } \beta 1, \text { COX-2 }\end{array}$ & $\begin{array}{l}\text { Angelica pubescens, Saposhnikovia divaricata, Ligusticum chuanxiong, } \\
\text { Gentiana macrophylla, Eucommia ulmoides, Angelicae sinensis, } \\
\text { Codonopsis pilosula, Asarum sieboldii, Glycyrrhiza uralensis }\end{array}$ \\
\hline 3 & ADAMTS4 & $\begin{array}{l}\text { Saposhnikovia divaricata, Ligusticum chuanxiong, Eucommia ulmoides, } \\
\text { Angelicae sinensis, Codonopsis pilosula, Radix rehmanniae preparata }\end{array}$ \\
\hline 4 & MMP-13, ER, VDR & Saposhnikovia divaricata, Glycyrrhiza uralensis \\
\hline 5 & COX-1, COX-2, ER, TGF- $\beta 1$ & $\begin{array}{l}\text { Ligusticum chuanxiong, Achyranthes bidentata, Angelicae sinensis, } \\
\text { Codonopsis pilosula, Glycyrrhiza uralensis }\end{array}$ \\
\hline
\end{tabular}

ADAMTS-4, Aggrecanase-1; MMP, matrix metalloproteinase; TNF- $\alpha$, tumor necrosis factor $\alpha$; iNOS, inducible nitric oxide synthase; COX, cyclooxygenase; CDK, cyclin-dependent kinase; HO, heme oxygenase; PPAR $\gamma$, peroxisome proliferator-activated receptor- $\gamma$; GSK-3 $\beta$, glycogen synthase kinase-3 $\beta$; ER, estrogen receptor; VDR, vitamin D nuclear receptor; TGF- $\beta 1$, transforming growth factor- $\beta$ type I

A

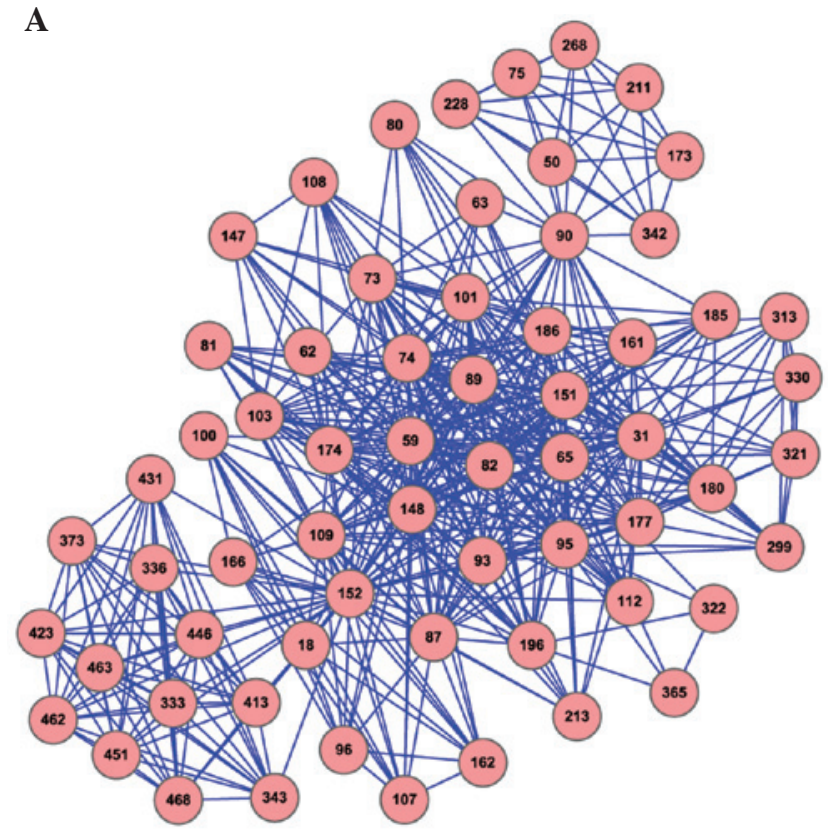

C

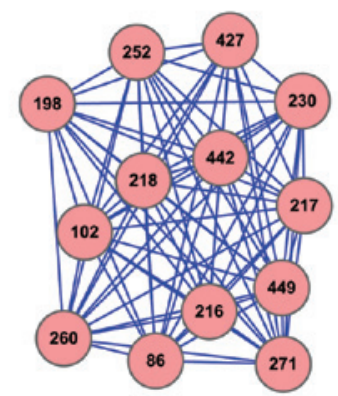

B

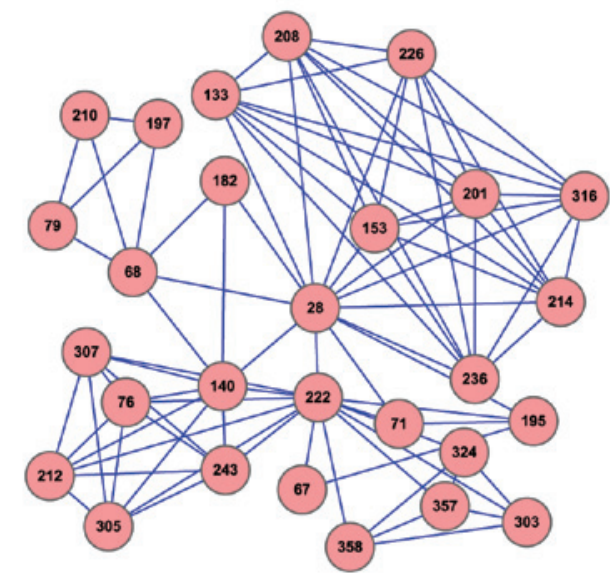

D

$\mathbf{E}$

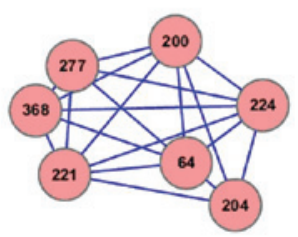

Figure 7. Subnetworks made up of highly interconnected regions. The pink nodes represent the DHJSD compounds. Clusters: (A) 1 , (B) 2 , (C) 3 , (D) 4 and (E) 5. DHJSD, Duhuo Jisheng Decoction. 
compounds of cluster 1 and 2 appeared to target multiple pathological processes and had combined synergistic effects. In addition, other combination patterns (clusters 3 and 4 or clusters 4 and 5) may produce alternative synergistic effects. Generally, multiple agents are used to achieve a maximum efficacy by aggressively attacking several targets simultaneously to exploit synergy and to minimize individual toxicity (32). Clinical observation has demonstrated that the effect of DHJSD on knee OA is superior to the efficacy of glucosamine sulfate and has good safety (33).Therefore, the DHJSD compounds with various mechanisms of anti-OA action interact principally in an additive or synergistic manner. This is easily explained by the fact that clusters (DHJSD) in various holistic combinations attack various targets or steps in the pathological process of OA.

In summary, in the present study, DHJSD was identified as diverse and drug-like in molecular composition. The DHJSD possessed abundant multi-target compounds and had significant potential for synergistic drug combinations against OA. The results demonstrated that potential synergy and polypharmacology properties exist in the curative mechanism of the TCM recipe.

\section{Acknowledgements}

This study was supported by the Developmental Fund of Chen Keji Integrative Medicine (CKJ2010032) and the National Natural Science Foundation of China (81202713).

\section{References}

1. Das SK and Farooqi A: Osteoarthritis. Best Pract Res Clin Rheumatol 22: 657-675, 2008.

2. McKellar G, Madhok R and Singh G: The problem with NSAIDs: what data to believe? Curr Pain Headache Rep 11: 423-427, 2007

3. Janga SC and Tzakos A: Structure and organization of drug-target networks: insights from genomic approaches for drug discovery. Mol Biosyst 5: 1536-1548, 2009.

4. Kitano H: A robustness-based approach to systems-oriented drug design. Nat Rev Drug Discov 6: 202-210, 2007.

5. Chen M, Feng W, Cao H, Zou L, Chen C, Baatrup A, Nielsen AB, Li H, Kassem M, Zou X and Bünger C: A traditional Chinese medicine formula extracts stimulate proliferation and inhibit mineralization of human mesenchymal stem cells in vitro. J Ethnopharmacol 125: 75-82, 2009.

6. Ma XH, Zheng CJ, Han LY, Xie B, Jia J, Cao ZW, Li YX and Chen YZ: Synergistic therapeutic actions of herbal ingredients and their mechanisms from molecular interaction and network perspectives. Drug Discov Today 14: 579-588, 2009.

7. Khanna D, Sethi G, Ahn KS, Pandey MK, Kunnumakkara AB, Sung B, Aggarwal A and Aggarwal BB: Natural products as a gold mine for arthritis treatment. Current Opinion in Rheumatol 7: 344-351, 2007.

8. Huh JE, Lee WI, Seo BK, Baek YH, Lee JD, Choi DY and Park DS: Gastroprotective and safety effects of WIN-34B, a novel treatment for osteoarthritis, compared to NSAIDs. J Ethnopharmacol 137: 1011-1017, 2011.

9. Zhang EQ: Bi Syndrome (Arthralgia Syndrome). J Tradit Chin Med 30:145-152, 2010.

10. Shu JS: Effect of Duhuo Jisheng Decoction on TNF- $\alpha$ and MMP-3 in Rabbit with Osteoarthritis. J Hubei UNIV TCM 14 3-5, 2012.

11. Chen W and Zhang YH: Influence of Duhuo Jisheng Decoction on cytokines from joint synovial fluid in patients with knee osteoarthritis. J Liaoning UNIV TCM 13: 216-217, 2011.
12. Lai JN, Chen HJ, Chen CC, Lin JH, Hwang JS and Wang JD: Duhuo jisheng tang for treating osteoarthritis of the knee: a prospective clinical observation. Chin Med 2: 4, 2007.

13. Xu X: New concepts and approaches for drug discovery based on traditional Chinese medicine. Drug Discov Today 3: 247-253, 2006.

14. Qiao X, Hou T, Zhang W, Guo S and Xu X: A 3D structure database of components from Chinese traditional medicinal herbs. J Chem Inf Comput Sci 42: 481-489, 2002.

15. Zhou JX, Xie GR and Yang XD: Handbook Of The Constituents In Chinese Herb Original Plants. 1st edition. Chemical Industry Press, Beijing, pp1165-1211, 2004.

16. Hassan M, Bielawski JP, Hempel JC and Waldman M: Optimization and visualization of molecular diversity of combinatorial libraries. Mol Divers 2: 64-74, 1996.

17. Dobson CM: Chemical space and biology. Nature 432: 824-828, 2004.

18. Zheng CS, Ye HZ, Xu XJ and Liu XX: Computational pharmacology study of tougu xiaotong granule in preventing and treating knee osteoarthritis. Chin J Integr Med 15: 371-376, 2009.

19. Zheng CS, Xu XJ and Ye HZ: Computational simulation of multi-target research on the material basis of Caulis sinomenii in treating osteoarthritis. Zhongguo Zhong Xi Yi Jie He Za Zhi 32: 375-379, 2012 (In Chinese).

20. Montes M, Braud E, Miteva MA, Goddard ML, Mondésert O, Kolb S, Brun MP, Ducommun B, Garbay C and Villoutreix BO: Receptor-based virual ligand screening for the identification of novel CDC25 phosphatase inhibitors. J Chem Inf Model 48: 157-165, 2008.

21. Smoot ME, Ono K, Ruscheinski J, Wang PL and Ideker T: Cytoscape 2.8: new features for data integration and network visualization. Bioinformatics 27: 431-432, 2011.

22. Gu J, Zhang H, Chen L, Xu S, Yuan G and Xu X: Drug-target network and polypharmacology studies of a Traditional Chinese Medicine for type II diabetes mellitus. Comput Biol Chem 35: 293-297, 2011.

23. Lipinski CA, Lombardo F, Dominy BW and Feeney PJ: Experimental and computational approaches to estimate solubility and permeability in drug discovery and development settings. Adv Drug Deliv Rev 46: 3-26, 2001.

24. Wishart DS, Knox C, Guo AC, Cheng D, Shrivastava S, Tzur D, Gautam B and Hassanali M: DrugBank: a knowledgebase for drugs, drug actions and drug targets. Nucleic Acids Res 36: D901-D906, 2008.

25. Garstang SV and Stitik TP: Osteoarthritis: epidemiology, risk factors, and pathophysiology. Am J Phys Med Rehabil 85 (11 Suppl): S2-S11, 2006.

26. Chan E, Tan M, Xin J, Sudarsanam S and Johnson DE: Interactions between traditional Chinese medicines and Western therapeutics. Curr Opin Drug Discov Devel 13: 50-65, 2010.

27. Chen WP, Tang JL, Bao JP, Hu PF, Shi ZL and Wu LD: Anti-arthritic effects of chlorogenic acid in interleukin-1 $\beta$ induced rabbit chondrocytes and a rabbit osteoarthritis model. Int Immunopharmacol 11: 23-28, 2011.

28. Gründemann C, Gruber CW, Hertrampf A, Zehl M, Kopp B and Huber R: An aqueous birch leaf extract of Betula pendula inhibits the growth and cell division of inflammatory lymphocytes. J Ethnopharmacol 136: 444-451, 2011.

29. He T, Yang LN and Zhao ZG: Embryogenesis of Gentiana straminea and assessment of genetic stability of regenerated plants using inter simple sequence repeat (ISSR) marker. Afr J Biotechnol 10: 7604-7610, 2011.

30. Li Y, Sato T, Metori K, Koike K, Che QM and Takahashi S: The promoting effects of geniposidic acid and aucubin in Eucommia ulmoides Oliver leaves on collagen synthesis. Biol Pharm Bull 21: 1306-1310, 1998

31. Brown JB and Okuno Y: Systems biology and systems chemistry: new directions for drug discovery. Chem Biol 19: 23-28, 2012.

32. Dhillon S: Naproxen/esomeprazole fixed-dose combination: for the treatment of arthritic symptoms and to reduce the risk of gastric ulcers. Drugs Aging 28: 237-248, 2011.

33. Yu JH and Zhang H: Duhuo Jisheng tang of knee osteoarthritis clinical observation. Chin J Exp Tradit Med Formulae 16: 215-217, 2010. 